\title{
Serum Correlation of Nesfatin-1 with Angiographic, Echocardiographic, and Biochemical Findings in Patients with Coronary Artery Disease
}

\author{
${ }^{1}$ Department of Cardiology, Faculty of Medicine, Ardabil University of Medical Sciences, Ardabil, Iran \\ 2 Faculty of Medicine, Ardabil University of Medical Sciences, Ardabil, Iran \\ ${ }^{3}$ Department of Physiology, Faculty of Medicine, Ardabil University of Medical Sciences, Ardabil, Iran \\ ${ }^{4}$ Applied Biomedical Research Center, Mashhad University of Medical Sciences, Mashhad, Iran
}

Afshin Nejati ${ }^{1}$, Hossein Doustkami ${ }^{1}$, Behzad Babapour ${ }^{1}$, Valeh Ebrahimoghlou ${ }^{2}$ and Mohammad Reza Aslani ${ }^{3,4}, *$

* Corresponding author: Mohammad Reza Aslani, Department of Physiology, Faculty of Medicine, Ardabil University of Medical Sciences, Ardabil, Iran. Tel: +984533262140; Email: mraslani105@yahoo.com, mr.aslani@arums.ac.ir

Received 2020 December 29; Revised 2021 January 11; Accepted 2021 February 16.

\begin{abstract}
Background: Nesfatin, as an adipokine, has been shown to have a number of metabolic effects associated with a variety of inflammatory diseases.

Objectives: The current study aimed at investigating the relationship of nesfatin-1 levels with biochemical, echocardiographic, and angiographic findings, especially with the epicardial fat thickness (EFT) in patients with coronary artery disease.

Methods: This cross-sectional study was conducted on 90 patients who were candidates for angiography. The patients were then divided into one control group (with normal angiography but a history of chest pain; $\mathrm{n}=30$ ), as well as stable-AP (with a diagnosis of stable angina pectoris; $n=30$ ) and acute-MI groups (with a diagnosis of acute myocardial infarction; $n=30$ ). Demographic, biochemical, angiographic, and echocardiographic parameters of all subjects were evaluated in this study.

Results: Serum levels of nesfatin-1 in acute-MI and stable-AP groups were significantly reduced, compared to the healthy subjects $(\mathrm{P}<0.001$ and $\mathrm{P}<0.01$, respectively); however, there was no significant difference between acute-MI and stable-AP groups. In addition, the findings demonstrated a negative relationship of nesfatin-1 with low-density lipoprotein, Gensini score, white blood cell, troponin T, EFT, and creatine kinase-MB (CK-MB). However, there was a positive association between nesfatin-1 and left ventricle ejection fraction (\%).

Conclusion: Decreased levels of nesfatin-1 in acute-MI and stable-AP groups as well as its association with several parameters, such as EFT, CK-MB, and Gensini score, may indicate the potential role of nesfatin-1 in the process of atherosclerosis, which requires further studies.
\end{abstract}

Keywords: Angiography, Coronary artery diseases, Epicardial fat thickness, Nesfatin

\section{Background}

Several pathological diseases indicate public health problems, such as metabolic syndrome, obesity, and cardiovascular diseases (CVD). The CVD is the major cause of death in most countries (1). Many factors, such as hyperlipidemia, smoking, family history, and blood pressure, have been reported in the occurrence and development of CVD (2). Although the exact mechanism of CVD is unclear, atherosclerosis has been suggested as a key mechanism (3). In the occurrence of coronary artery atherosclerosis, obesity is the fundamental risk factor. Adipose tissue plays an important role in most inflammatory diseases, such as CVD, asthma, and chronic obstructive pulmonary disease (COPD) by releasing a variety of mediators and cytokines called adipokines (4-7).

Nesfatin- 1 is a peptide with 82 amino acids that is produced as a result of the proteolysis of nucleobindin 2 (8). Furthermore, it is known as the primary satiety hormone secreted from the arachnoid, paraventricular, supraventricular, and hypothalamic nuclei $(8,9)$. Intraventricular or intraperitoneal injection of the peptide into mice showed a reduced appetite (10). Recently, the studies have revealed a close association of nesfatin- 1 with CAD, mental disorders, neurological disease, diabetes, and polycystic ovaries syndrome (11-15). Reduced levels of nesfatin-1 have been reported in patients with acute myocardial infarction (acute-MI) as well as in cardiac tissue under ischemia/reperfusion injury (16). Except for one study (17), most evidence has shown reduced nesfatin-1 levels in patients with CAD (18). Similar findings have been obtained by Kuyumcu et al. regarding the reduced levels of nesfatin-1 in slow coronary flow patients (19).

There have been many studies recently on the role of epicardial fat tissue (EFT) in metabolic and cardiovascular diseases. It has been shown that the EFT can affect cardiovascular function by synthesizing and secreting adipokines and biologically active factors (20). Under physiological conditions, the EFT acts as a buffer to protect the heart by absorbing free fatty acids. The EFT also acts as a source to supply energy to the heart by storing fat when the heart needs a lot of energy (21). However, the endocrine role of EFT in physiological and pathological conditions is so important that EFT can express and synthesize a large number of cytokines, pro-inflammatory and antiinflammatory adipokines, vascular mediators, and growth factors $(21,22)$. Increased thickness 
of epicardial adipose tissue has been shown to release autocrine/vasocrine/endocrine manner of inflammatory mediators, such as interleukin-6 (IL-6), tissue necrosis factor (TNF- $\alpha$ ), and adipocytokines causing coronary atherosclerosis $(22,23)$.

Despite the contradictory results regarding the serum levels of nesfatin- 1 in CAD patients, there is no study on the relationship between nesfatin-1 and echocardiographic findings, especially with EFT.

\section{Objectives}

Therefore, the current study aimed to test the levels of nesfatin-1 in acute-MI and stable angina pectoris (stable-AP) patients in comparison with the control subjects; moreover, it was attempted to investigate its association with angiographic, echocardiographic, biochemical, and demographic findings.

\section{Methods}

The findings of this study were part of the results of a previous study that was conducted from April 2018 to July 2019 in Imam Khomeini Educational and Clinical Hospital, Ardabil, Iran (24). In a crosssectional comparative study, out of 120 patients candidate for angiography, 90 individuals who met the inclusion criteria were included in this study. The patients were then divided into one control group (suffering from chest pain complaints and no angiographic findings; $n=30$ ), and two groups of clinically diagnosed with CAD (acute-MI; $n=30$; stable-AP; $n=30$ ).

On the other hand, the patients with a history of MI, autoimmune diseases, myocarditis, chronic respiratory diseases, valvular heart diseases, serious heart failure, acute or chronic infectious diseases, cancer, steroid therapy, pericardial effusion, hepatitis, chronic renal failure, and poor echocardiographic imaging were excluded from the study. The inclusion criteria in the acute-MI group were changes in biochemical tests, such as an increase in creatine kinase-MB (CK-MB) and troponin $\mathrm{T}$ serum levels or electrocardiogram (ECG) changes, such as an increase in the ST-segment based on the diagnosis of acute-MI. Regarding the stable-AP group, the inclusion criteria were based on changes in the ECG during the exercise test with a feeling of discomfort in the chest.

Demographic information, systolic and diastolic blood pressure, associated comorbidities, waist-hip ratio (WHR), cardiovascular risk factors, body mass index (BMI), and medical history of all subjects were collected in this study. Furthermore, hypertension, hyperlipidemia, and type 2 diabetes were defined based on previous studies (24).

It should be mentioned that serum samples of the subjects from the previous study were used for biochemical measurements. Moreover, serum CK-MB, nesfatin-1, and troponin $\mathrm{T}$ levels were measured using enzyme-linked immunosorbent assay kits and standard methods.

All echocardiographic and angiographic assessments of the study subjects have been fully described previously (24). Briefly, the measured echocardiographic parameters included tricuspid lateral annular systolic velocity, annular plane systolic excursion, mitral valve lateral annular systolic velocity, mitral valve septal annular systolic velocity, left ventricular ejection fraction (LVEF), and EFT.

The standard Judkins technique was used in angiography; moreover, 5 or 6 Fr catheters were performed on radial or femoral arteries on either the right or left coronary. The modified Gensini score was used to measure the severity of the CAD (25). This index is one of the valid methods for measuring the severity of coronary artery stenosis. In this index, eight branches of coronary arteries are scored based on the degree of stenosis, and the stenosis scores ranged from $1(1 \%-49 \%), 2$ (50\%-75\%), 3 (75\%$99 \%)$, to $4(100 \%)$. In addition, based on the type of artery, coefficient 20 (Right coronary artery, Left anterior descending artery, and Left circumflex), coefficient 10 (OM1, Posterior descending artery, and D1), and coefficient 5 (Left main and S1) were applied in this method. Finally, the Gencini score was estimated between 0 and 400 based on the severity of the stenosis coefficient score.

\subsection{Statistical Analysis}

The obtained data were analyzed in SPSS software (version 21) through the Kolmogorov-Smirnov test to measure the normal distribution of the results. The data with normal distribution were reported as mean \pm SD; otherwise, they were presented as median and the $25^{\text {th }}-75^{\text {th }}$ percentiles. Furthermore, a comparison between groups was performed using the Kruskal-Wallis test (with Mann-Whitney post hoc test) or ANOVA test (with Tukey Kemar post hoc test). To adjust the results with smoking status, age, WHR, and BMI, the General linear modeling function analysis was utilized in this study. Spearman rank order or Pearson's test was also employed to determine the correlation coefficients. A p-value less than 0.05 was considered statistically significant.

\section{Results}

\subsection{Demographic and clinical findings}

Table 1 indicates the clinical and demographic characteristics of the study groups. The results revealed a significant difference among the study groups in terms of the mean age $(\mathrm{P}<0.01)$, WHR $(\mathrm{P}<0.01)$, systolic and diastolic blood pressure $(\mathrm{P}<0.001$ and $\mathrm{P}<0.01$, respectively), total cholesterol (TC) $(\mathrm{P}<0.05)$, low-density lipoprotein cholesterol (LDL-C) $(\mathrm{P}<0.001)$, CK-MB $(\mathrm{P}<0.001)$, white blood 


\begin{tabular}{|c|c|c|c|c|}
\hline variable & $\begin{array}{c}\text { Control } \\
(\mathrm{n}=30)\end{array}$ & $\begin{array}{c}\text { Stable-AP } \\
(n=30)\end{array}$ & $\begin{array}{c}\text { Acute-MI } \\
(n=30)\end{array}$ & P-value \\
\hline Age (year) & $61.66 \pm 13.08$ & $63.86 \pm 8.70$ & $54.90 \pm 8.92$ & 0.004 \\
\hline BMI $\left(\mathrm{kg} / \mathrm{m}^{2}\right)$ & $26.09 \pm 3.08$ & $26.86 \pm 4.53$ & $27.86 \pm 3.68$ & 0.205 \\
\hline Waist circumference (cm) & $94.16 \pm 5.36$ & $97.20 \pm 9.81$ & $98.83 \pm 6.70$ & 0.057 \\
\hline Hip circumference (cm) & $101.23 \pm 4.68$ & $100.43 \pm 9.11$ & $102.50 \pm 8.11$ & 0.567 \\
\hline Waist-hip ratio & $0.93 \pm 0.03$ & $0.96 \pm 0.03$ & $0.96 \pm 0.06$ & 0.002 \\
\hline Systolic blood pressure (mmHg) & $123.50 \pm 12.94$ & $137.80 \pm 17.06$ & $121.43 \pm 14.28$ & 0.000 \\
\hline Diastolic blood pressure (mmHg) & $79.00 \pm 7.47$ & $83.33 \pm 10.53$ & $72.33 \pm 15.75$ & 0.002 \\
\hline Heart rate (bpm) & $72.73 \pm 10.04$ & $73.83 \pm 7.48$ & $77.96 \pm 11.42$ & 0.098 \\
\hline $\mathrm{SpO}_{2}(\%)$ & $95.30 \pm 1.17$ & $94.40 \pm 1.32$ & $95.13 \pm 2.02$ & 0.064 \\
\hline Smoking, n (\%) & $12(40 \%)$ & $12(40 \%)$ & $15(50 \%)$ & 0.440 \\
\hline Hypertension, n (\%) & $13(43.3 \%)$ & $14(46.7 \%)$ & $8(26.7 \%)$ & 0.190 \\
\hline Diabetes, n (\%) & $3(10 \%)$ & $8(26.7 \%)$ & $6(20 \%)$ & 0.328 \\
\hline Hyperlipedemia, n (\%) & $11(36.7 \%)$ & $19(63.3 \%)$ & $8(26.7 \%)$ & 0.439 \\
\hline Familiar heart disease, n (\%) & $14(46.7 \%)$ & $14(46.7 \%)$ & $13(43.3 \% 0$ & 0.798 \\
\hline TC (mg/dL) & $154.76 \pm 20.62$ & $169.96 \pm 29.56$ & $174.50 \pm 29.67$ & 0.015 \\
\hline$T G(\mathrm{mg} / \mathrm{dL})$ & $113.33 \pm 43.87$ & $128.20 \pm 52.74$ & $128.30 \pm 43.03$ & 0.366 \\
\hline HDL-C (mg/dL) & $44.50 \pm 7.20$ & $41.16 \pm 7.71$ & $41.33 \pm 7.05$ & 0.145 \\
\hline LDL-C (mg/dL) & $81.00 \pm 18.08$ & $99.93 \pm 19.15$ & $107.63 \pm 21.72$ & 0.000 \\
\hline FBG (mg/dL) & $98.06 \pm 9.79$ & $102.43 \pm 9.16$ & $101.20 \pm 9.26$ & 0.186 \\
\hline BUN (mg/dL) & $36.70 \pm 9.38$ & $42.66 \pm 22.44$ & $44.70 \pm 29.28$ & 0.346 \\
\hline $\operatorname{Cr}(\mathrm{mg} / \mathrm{dL})$ & $1.24 \pm 0.21$ & $1.27 \pm 0.30$ & $1.31 \pm 0.40$ & 0.666 \\
\hline Uric acid (mg/dL) & $5.51 \pm 1.37$ & $5.86 \pm 1.77$ & $6.14 \pm 1.78$ & 0.341 \\
\hline Hemoglobin $(\mathrm{g} / \mathrm{dL})$ & $14.46 \pm 1.78$ & $14.25 \pm 1.21$ & $14.65 \pm 2.33$ & 0.705 \\
\hline WBC $\left(10^{3} / \mathrm{mm}^{3}\right)$ & $7.34 \pm 1.81$ & $8.69 \pm 1.25$ & $9.33 \pm 1.80$ & 0.000 \\
\hline Platelet $\left(10^{3} / \mathrm{mm}^{3}\right)$ & $196.1 \pm 53.75$ & $224.56 \pm 47.77$ & $230.76 \pm 82.12$ & 0.081 \\
\hline CK-MB (ng/mL) & $2.74 \pm 0.47$ & $3.77 \pm 1.31$ & $34.43 \pm 11.45$ & 0.000 \\
\hline HsTnT (ng/L) & $2.66 \pm 2.02$ & $10.73 \pm 5.10$ & $34.68 \pm 10.99$ & 0.000 \\
\hline Gensini score & Not done & $31.66 \pm 14.22$ & $47.33 \pm 15.96$ & 0.000 \\
\hline
\end{tabular}

Data are presented as the mean \pm SD (or median \pm IQR) and percentage (\%).

Stable-AP: stable angina pectoris, acute-MI: acute myocardial infarction, BP: blood pressure, BMI: body mass index, HsTnT: highsensitivity troponin $\mathrm{T}$ TC: Total cholesterol, $\mathrm{SpO}_{2}: \mathrm{O}_{2}$ saturation, TG: triglyceride, HDL: high-density lipoprotein, LDL: low-density lipoprotein, WBC: white blood cell, BUN: blood urea nitrogen, FBG: Fasting blood glucose, Cr: creatinine, CK-MB: creatine kinase-MB

cells (WBC) $(\mathrm{P}<0.001)$, troponin $\mathrm{T}(\mathrm{P}<0.001)$, and Gensini score $(\mathrm{P}<0.001)$. The mean age of the acuteMI patients was significantly lower than that in the stable-AP and control subjects. Moreover, the acuteMI group obtained higher values of the study parameters, such as LDL-C, WBC, TC, CK-MB, troponin $\mathrm{T}$, and Gensini scores, compared to the stable-AP and control groups. On the other hand, systolic and diastolic blood pressure was higher in the stable-AP group, compared to the control group. As can be observed in Table 1, there is no significant difference among the study groups regarding the BMI, triglyceride, fasting blood glucose, uric acid, heart rate, familiar heart disease history, hypertension, smoking status, diabetes mellitus, hyperlipidemia, high-density lipoprotein cholesterol, hemoglobin, blood urea nitrogen, platelet, and creatinine.

The findings obtained from the serum levels of nesfatin- 1 demonstrated that acute-MI $(17.22 \pm 2.75)$ and stable-AP $(19.72 \pm 3.60)$ groups had lower serum levels of nesfatin-1, compared to the control subjects (25.51 $\pm 10.11 ; \quad \mathrm{P}<0.001$ and $\mathrm{P}<0.01$, respectively); however, no significant difference was observed between stable-AP and acute-MI groups in this regard (Figure 1 a). Furthermore, the adjustment of nesfatin1 results for age, WHR, BMI, and group showed that there was still a difference regarding the serum levels of nesfatin- 1 in stable-AP and acute-MI groups with the control group $(\mathrm{P}<0.001$ for both). Interestingly, serum levels of adjusted-nesfatin-1 also revealed a significant difference between the acute-MI group and the stable-AP group $(\mathrm{P}<0.001$; Figure $1 \mathrm{~b})$.

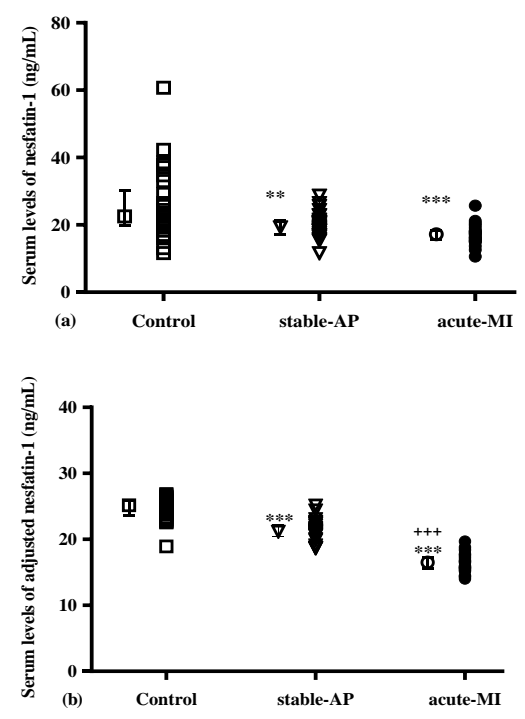

Figure 1. Individual values and mean $\pm \mathrm{SD}$ of serum levels of (a): nesfatin-1, and (b): adjusted nesfatin-1 for age, BMI, WHR, and group in the study groups. For statistical differences between the control group and other groups: ***; $\mathrm{P}<0.001$. For statistical differences between stable-AP with acute-MI: +++; $\mathrm{P}<0.001$. 


\begin{tabular}{|c|c|c|c|c|}
\hline Variables & Control & stable-AP & acute-MI & P-value \\
\hline LVEF (\%) & $52.83 \pm 7.73$ & $42.66 \pm 7.84$ & $35.40 \pm 9.46$ & 0.000 \\
\hline$e^{\prime}$ Septal $(\mathrm{cm} / \mathrm{s})$ & $6.73 \pm 1.36$ & $6.25 \pm 1.77$ & $6.97 \pm 1.95$ & 0.265 \\
\hline$e^{\prime}$ Lateral $(\mathrm{cm} / \mathrm{s})$ & $9.78 \pm 2.20$ & $9.61 \pm 2.44$ & $9.40 \pm 2.55$ & 0.832 \\
\hline TAPSE (mm) & $19.55 \pm 3.36$ & $17.77 \pm 3.17$ & $18.00 \pm 3.21$ & 0.077 \\
\hline TV TDI $(\mathrm{cm} / \mathrm{s})$ & $11.61 \pm 1.57$ & $12.16 \pm 1.80$ & $11.34 \pm 2.12$ & 0.222 \\
\hline EFT (mm) & $4.40 \pm 0.77$ & $6.11 \pm 1.01$ & $7.81 \pm 1.17$ & 0.000 \\
\hline EFT (adjusted), (mm)a & $4.46 \pm 0.12$ & $6.12 \pm 0.07$ & $7.81 \pm 0.13$ & 0.000 \\
\hline
\end{tabular}

Data are presented as mean \pm SD

a Mean \pm SD by a general linear model with adjustment for age, BMI, WHR, and group.

Echocardiographic findings showed that LVEF (\%) was significantly lower in the acute-MI group

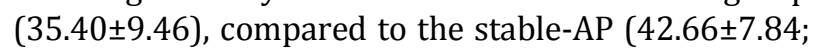
$\mathrm{P}<0.01)$ and control $(52.83 \pm 7.73$; $\mathrm{P}<0.001)$ groups (Table 2). Moreover, the LVEF (\%) was significantly lower in the stable-AP group, compared to the control group $(\mathrm{P}<0.001)$. Furthermore, the EFT findings indicated that the EFT in the acute-MI $(7.81 \pm 1.17)$ and stable-AP $(6.11 \pm 1.01)$ groups was significantly higher than that in the control group $(4.40 \pm 0.77 ; \mathrm{P}<0.001$ for both). In addition, the EFT in the acute-MI group was significantly higher than that in the stable-AP group $(\mathrm{P}<0.001$; Figure 2a). The adjustment of the results for WHR, BMI, age, and group revealed that there was again a statistically significant difference regarding EFT $(\mathrm{P}<0.001$; Figure $2 b)$.

\subsection{Association of nesfatin-1 with study parameters}

According to the results of Pearson correlation
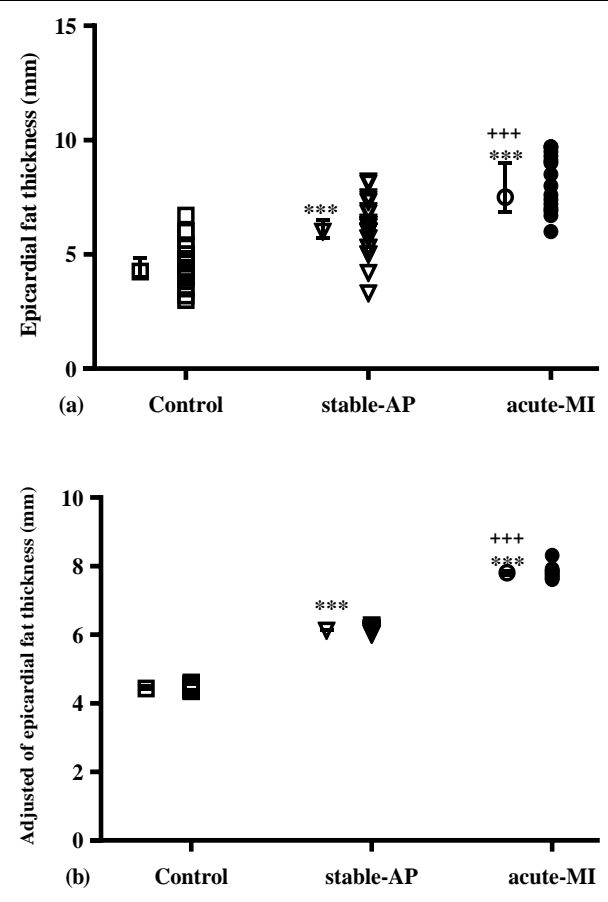

Figure 2. Individual values and mean \pm SD of the epicardial fat thickness of (a) baseline, and (b) adjusted for age, BMI, WHR, and group in the study groups. For statistical differences between the control group and other groups: ${ }^{* * *}$; $\mathrm{P}<0.001$. For statistical differences between stable-AP with acute-MI: +++ P $<0.001$. analysis, nesfatin- 1 showed a negative significant relationship with CK-MB, LDL-C, troponin T, WBC, Gensini score, and EFT. However, it has a positive relationship with LVEF (\%) (Table 3). On the other hand, no significant association was found between nesfatin- 1 and other parameters studied.

\begin{tabular}{|c|c|c|}
\hline \multirow{2}{*}{ Variables } & \multicolumn{2}{|c|}{ Nesfatin-1 } \\
\hline & $\mathbf{r}$ & P-value \\
\hline Age & 0.043 & 0.686 \\
\hline BMI & 0.050 & 0.639 \\
\hline WHR & -0.193 & 0.068 \\
\hline Systolic blood pressure & 0.004 & 0.972 \\
\hline Diastolic blood pressure & 0.062 & 0.560 \\
\hline Heart rate & -0.073 & 0.495 \\
\hline Sp02 & 0.044 & 0.682 \\
\hline FBG & 0.104 & 0.328 \\
\hline T-cholesterol & -0.207 & 0.050 \\
\hline Triglyceride & -0.163 & 0.125 \\
\hline HDL-C & 0.056 & 0.600 \\
\hline LDL-C & -0.313 & 0.003 \\
\hline CK-MB (ng/mL) & -0.316 & 0.002 \\
\hline Troponin T (ng/mL) & -0.377 & 0.000 \\
\hline WBC $\left(10^{3} / \mathrm{mm}^{3}\right)$ & -0.319 & 0.002 \\
\hline Hemoglobin (g/dL) & -0.055 & 0.604 \\
\hline Platelet $\left(10^{3} / \mathrm{mm}^{3}\right)$ & -0.190 & 0.073 \\
\hline Gensini score & -0.416 & 0.000 \\
\hline LVEF (\%) & 0.340 & 0.001 \\
\hline$e^{\prime} S e p t a l(\mathrm{~cm} / \mathrm{s})$ & -0.031 & 0.771 \\
\hline$e^{\prime}$ Lateral $(\mathrm{cm} / \mathrm{s})$ & -0.058 & 0.587 \\
\hline TAPSE (mm) & 0.177 & 0.095 \\
\hline TV TDI $(\mathrm{cm} / \mathrm{s})$ & -0.086 & 0.419 \\
\hline EFT (mm) & -0.363 & 0.000 \\
\hline
\end{tabular}

FBG: fasting blood glucose, WHR: waist-hip ratio, BMI: body mass index, LDL-C: low-density lipoprotein-cholesterol, CKMB: creatine kinase-MB, SpO2: $\mathrm{O}_{2}$ saturation, HDL-C: highdensity lipoprotein-cholesterol, WBC: white blood cell, LVEF: left ventricular ejection fraction, TAPSE: tricuspid annular plane systolic excursion, septal e': mitral valve septal annular systolic velocity, Lateral e': mitral valve lateral annular systolic velocity, TV TDI: tricuspid lateral annular systolic velocity, EFT: epicardial fat thickness

\section{Discussion}

The results of the current study revealed that nesfatin-1 serum levels significantly were lower in stable-AP and acute-MI patients, compared to the control group. Furthermore, there was a negative correlation between serum nesfatin-1 levels and some study parameters, such as EFT, troponin T, Gensini score, LDL-C, and CK-MB.

Evidence from animal and human studies has 
shown that adipose tissue (AP) plays a critical role in some chronic inflammatory diseases, such as cardiovascular disease, rheumatoid arthritis, asthma, and COPD $(5,6,24)$. Moreover, the AP is of utmost importance in the pathogenesis of the disease by activating autocrine/paracrine/endocrine pathways to release a variety of chemical mediators, such as adipolin, visfatin, leptin, fatty acid-binding protein-4, and nesfatin-1 $(6,24,26)$. Nesfatin-1 has been initially recognized as a satiety hormone, as its intracerebroventricular injection in the animal study led to decreased food intake (8).

Dai et al. reported that patients with acute-MI had lower plasma levels of nesfatin-1, compared to controls, which were in line with the results of the current study (11). Our study also revealed low serum nesfatin-1 levels in patients with stable angina patients, compared to controls. It is worth mentioning that after adjusting the results of nesfatin-1 for age, BMI, smoking history, and group, a significant difference was also demonstrated between acute-MI and stable-AP groups. Moreover, further analysis indicated a negative correlation of serum levels of nesfatin-1 with Gensini score, LDL-C, CK-MB, troponin $\mathrm{T}$, and WBC. Specific markers of myocardial cell damage that are increased in acute-MI include CK-MB and troponin T (27). Dai et al. showed reduced plasma nesfatin-1 levels in patients with acute-MI. They also reported a significant negative correlation between nesfatin-1 plasma levels and inflammatory factors, such as neutrophil percentage and C-reactive protein (11). Elevated levels of proinflammatory mediators, such as TNF- $\alpha$, IL-6, and IL-1 $\beta$, have been reported in both serum and heart tissue during MI (28). Inflammation is one of the most important mechanisms of myocardial infarction. The antiinflammatory effects of nesfatin-1 have been reported in some studies (11); accordingly, at least in part, reduced levels of nesfatin-1 in patients with acute-MI may indicate its role in the pathology of the disease, which requires further studies. In the same line, Tasatargil et al., in an animal model of isoproterenolinduced MI, reported the anti-inflammatory effects of nesfatin-1 in preventing histopathological changes in cardiac tissue and increasing troponin $\mathrm{T}$ (28).

Various mechanisms for the anti-inflammatory and protective nesfatin-1 effects have been reported so far. Animal studies have shown the antiinflammatory effects of nesfatin-1 on hyperglycemic and colitis models by inhibiting apoptosis and neutrophil recruitment $(29,30)$. Additionally, treatment with nesfatin-1 has been shown to reduce acute renal I/R damage through the inhibition of cellular apoptosis and oxidative stress (28). Regarding the cardioprotective effects of nesfatin-1, it has been suggested to reduce the inflammatory factors or increase cell survival by reducing neutrophils and inactivating the caspase cascade, pro-survival kinase protein kinase C (PKC)- $\varepsilon$, mitochondrial potassium-dependent ATP channel, and PI3K-Akt-GSK-3 $\beta$ pathway $(28,31,32)$.

This study investigated for the first time the negative association between EFT and serum nesfatin-1 levels. The results also showed a positive relationship between nesfatin-1 and LVEF. The EFT has been proposed as an independent measure of visceral fat and is higher in people with higher WHR. (20). Human and animal studies reveal that EFT is a significant source of anti-inflammatory, proatherogenic, adipokine, and proinflammatory agents, such as leptin, adiponectin, visfatin, resistin, omentin, monocyte chemoattractant protein-1, TNF- $\alpha$, IL-6, nerve growth factor, plasminogen activator inhibitor1 , and angiotensinogen $(33,34)$. It was found that the amount of EFT in echocardiography was higher in patients with CAD, compared to the healthy individuals (20). Although the exact mechanism of EFT in the pathogenesis of patients with CAD is unknown, EFT appears to be involved in the pathogenesis of atherosclerosis with paracrine/ endocrine effects (35). In a previous study, a relationship was shown between EFT and some parameters, such as the Gensini score, LVEF, and WBC (24). In general, the EFT is thought to play a key role in the pathogenesis of the disease in patients with CAD that requires further studies.

Regarding the limitations of this study, one can refer to the lack of further evaluation of gender differences due to the presence of no female gender. Moreover, since various studies have shown the protective effects of nesfatin-1 against inflammatory cytokines, the direct effects of nesfatin-1 on inflammatory cytokines appear to be appropriate. Finally, the sample size in this study was moderate, and a larger sample is required to understand the causal relationship between nesfatin-1 and CAD.

\section{Conclusion}

In conclusion, the current study showed a negative association between nesfatin-1 serum level and EFT in patients with acute-MI and stable-AP for the first time. Although the exact mechanism of pathogenesis in patients with CAD is not known, adipose tissue plays a key role in its pathogenesis by releasing adipokines. Despite the obvious antiinflammatory effects of nesfatin-1 in previous studies, more studies are required to be carried out to show the causal link in patients with CAD.

\section{Acknowledgments}

The authors would like to thank all the laboratory staff of Imam Khomeini Hospital, Ardabil, Iran.

\section{Footnotes}

Authors' Contribution: Mohammad Reza Aslani, 
Afshin Nejati, Hossein Doustkami, and Behzad Babapour: literature search, proposal writing, data collection, analysis of data, interpretation of data, manuscript preparation, and review of the manuscript. Valeh Ebrahimoghlou: data collection, analysis, and draft preparation.

Conflict of Interests: The authors have no conflict of interest to declare.

Ethical Approval: The study was approved by the Ethics Committee of Ardabil University of Medical Sciences, Ardabil, Iran (No. IR.ARUMS.REC.1397.302). Funding/Support: This study was funded by the Ardabil University of Medical Sciences, Ardabil, Iran.

Financial Disclosure: None declared.

Informed Consent: All of the study participants signed the written consent forms.

\section{References}

1. Sanchis-Gomar F, Perez-Quilis C, Leischik R, Lucia A. Epidemiology of coronary heart disease and acute coronary syndrome. Ann Transl Med. 2016;4(13):256. doi: 10.21037/ atm.2016.06.33. [PubMed: 27500157].

2. Veeranna V, Pradhan J, Niraj A, Fakhry H, Afonso L. Traditional cardiovascular risk factors and severity of angiographic coronary artery disease in the elderly. Prev Cardiol. 2010; 13(3):135-40. doi: 10.1111/j.1751-7141.2009.00062.x. [PubMed: 20626669].

3. Legein B, Temmerman L, Biessen EA, Lutgens E. Inflammation and immune system interactions in atherosclerosis. Cell Mol Life Sci. 2013;70(20):3847-69. doi: 10.1007/s00018-0131289-1. [PubMed: 23430000].

4. Keyhanmanesh R, Alipour MR, Ebrahimi H, Aslani MR. Effects of diet-induced obesity on tracheal responsiveness to methacholine, tracheal visfatin level, and lung histological changes in ovalbumin-sensitized female Wistar rats. Inflammation. 2018;41(3):846-58. doi: 10.1007/s10753-0180738-2. [PubMed: 29380115].

5. Akhavanakbari G, Babapour B, Alipour MR, Keyhanmanesh R, Ahmadi M, Aslani MR. Effect of high fat diet on NF-кB microRNA146a negative feedback loop in ovalbumin-sensitized rats. BioFactors. 2019;45(1):75-84. doi: 10.1002/biof.1466. [PubMed: 30521085].

6. Aslani MR, Ghazaei Z, Ghobadi H. Correlation of serum fatty acid binding protein-4 and interleukin-6 with airflow limitation and quality of life in stable and acute exacerbation of COPD. Turk J Med Sci. 2020;50(2):337-45. doi: 10.3906/sag1909-9. [PubMed: 31905499].

7. Kuyumcu MS, Kuyumcu A, Yayla C, Özbay MB, Ünal S, Açar B, et al. The relationship between nesfatin-1 levels and SYNTAX score in patients with non-ST segment elevation myocardial infarction. Acta Cardiol Sin. 2018;34(5):386-93. doi: 10.6515/ACS.201809_34(5).20180423A. [PubMed: 30271088].

8. Oh S, Shimizu H, Satoh T, Okada S, Adachi S, Inoue K, et al. Identification of nesfatin-1 as a satiety molecule in the hypothalamus. Nature. 2006;443(7112):709-12. doi: 10.1038/ nature05162. [PubMed: 17036007].

9. Yamawaki H. Vascular effects of novel adipocytokines: focus on vascular contractility and inflammatory responses. Biol Pharm Bull. 2011;34(3):307-10. doi: 10.1248/bpb.34.307. [PubMed: 21372376].

10. Shimizu H, Oh-i S, Hashimoto K, Nakata M, Yamamoto S, Yoshida $\mathrm{N}$, et al. Peripheral administration of nesfatin-1 reduces food intake in mice: the leptin-independent mechanism. Endocrinology. 2009;150(2):662-71. doi: 10.1210/en.20080598. [PubMed: 19176321].

11. Dai H, Li X, He T, Wang Y, Wang Z, Wang S, et al. Decreased plasma nesfatin-1 levels in patients with acute myocardial infarction. Peptides. 2013;46:167-71. doi: 10.1016/j.peptides.2013.06.006.
[PubMed: 23806888].

12. Li QC, Wang HY, Chen X, Guan HZ, Jiang ZY. Fasting plasma levels of nesfatin-1 in patients with type 1 and type 2 diabetes mellitus and the nutrient-related fluctuation of nesfatin-1 level in normal humans. Regul Pept. 2010;159(1-3):72-7. doi: 10.1016/j.regpep.2009.11.003. [PubMed: 19896982].

13. Deniz R, Gurates B, Aydin S, Celik H, Sahin I, Baykus Y, et al Nesfatin-1 and other hormone alterations in polycystic ovary syndrome. Endocrine. 2012;42(3):694-9. doi: 10.1007/s12020012-9638-7. [PubMed: 22367584].

14. Ari M, Ozturk OH, Bez Y, Oktar S, Erduran D. High plasma nesfatin-1 level in patients with major depressive disorder. Prog Neuropsychopharmacol Biol Psychiatry. 2011;35(2):497500. doi: 10.1016/j.pnpbp.2010.12.004. [PubMed: 21163317].

15. Aydin S, Dag E, Ozkan Y, Erman F, Dagli AF, Kilic N, et al. Nesfatin-1 and ghrelin levels in serum and saliva of epileptic patients: hormonal changes can have a major effect on seizure disorders. Mol Cell Biochem. 2009;328(1-2):49-56. doi: 10.1007/s11010-009-0073-x. [PubMed: 19262995].

16. Feijóo-Bandín $S$, Rodríguez-Penas D, García-Rúa V, MosqueraLeal A, González-Juanatey JR, Lago F. Nesfatin-1: a new energyregulating peptide with pleiotropic functions. Implications at cardiovascular level. Endocrine. 2016;52(1):11-29. doi: 10.1007/s12020-015-0819-z. [PubMed: 26662184].

17. Ibe S, Kishimoto Y, Niki H, Saita E, Umei T, Miura K, et al. Associations between plasma nesfatin-1 levels and the presence and severity of coronary artery disease. Heart Vessels. 2019;34(6):965-70. doi: 10.1007/s00380-018-01328-3. [PubMed: 30599062].

18. Angelone T, Rocca C, Pasqua T. Nesfatin-1 in cardiovascular orchestration: From bench to bedside. Pharmacol Res. 2020;156:104766. doi: 10.1016/j.phrs.2020.104766. [PubMed: 32201244].

19. Kuyumcu MS, Kuyumcu A, Yayla Ç, Özbay MB, Alagöz M, Ünal S, et al. Nesfatin-1 levels in patients with slow coronary flow. Kardiol Pol. 2018;76(2):401-5. doi: 10.5603/KP.a2017.0210. [PubMed: 29131290].

20. Iacobellis G, Willens HJ. Echocardiographic epicardial fat: a review of research and clinical applications. J Am Soc Echocardiogr. 2009;22(12):1311-9. doi: 10.1016/j.echo.2009.10.013. [PubMed: 19944955].

21. Iacobellis G, Bianco AC. Epicardial adipose tissue: emerging physiological, pathophysiological and clinical features. Trends Endocrinol Metab. 2011;22(11):450-7. doi: 10.1016/j.tem. 2011.07.003. [PubMed: 21852149].

22. Iozzo P. Myocardial, perivascular, and epicardial fat. Diabetes Care. 2011;34(Suppl 2):S371-9. doi: 10.2337/dc11-s250. [PubMed: 21525485].

23. Sacks HS, Fain JN. Human epicardial adipose tissue: a review. Am Heart J. 2007;153(6):907-17. doi: 10.1016/j.ahj.2007.03.019. [PubMed: 17540190].

24. Babapour B, Doustkami H, Avesta L, Moradi A, Saadat S Piralaei K, et al. Correlation of serum adipolin with epicardial fat thickness and severity of coronary artery diseases in acute myocardial infarction and stable angina pectoris patients. Med Princ Pract. 2020;In Press. doi: 10.1159/000508834. [PubMed: 32438366].

25. Sullivan DR, Marwick TH, Freedman SB. A new method of scoring coronary angiograms to reflect extent of coronary atherosclerosis and improve correlation with major risk factors. Am Heart J. 1990;119(6):1262-7. doi: 10.1016/s00028703(05)80173-5. [PubMed: 1972310].

26. Aslani MR, Keyhanmanesh R, Khamaneh AM, Abbasi MM, Fallahi M, Alipour MR. Tracheal overexpression of IL-1 $\beta$, IRAK1 and TRAF- 6 mRNA in obese-asthmatic male Wistar rats. Iran J Basic Med Sci. 2016;19(4):350-7. [PubMed: 27279977].

27. Katus HA, Remppis A, Neumann FJ, Scheffold T, Diederich KW, Vinar $G$, et al. Diagnostic efficiency of troponin $T$ measurements in acute myocardial infarction. Circulation. 1991;83(3):902-12. doi: 10.1161/01.cir.83.3.902. [PubMed: 1999039].

28. Tasatargil A, Kuscu N, Dalaklioglu S, Adiguzel D, Celik-Ozenci C, Ozdem S, et al. Cardioprotective effect of nesfatin-1 against isoproterenol-induced myocardial infarction in rats: role 
of the Akt/GSK-3 $\beta$ pathway. Peptides. 2017;95:1-9. doi: 10.1016/j.peptides.2017.07.003. [PubMed: 28720397].

29. Solmaz A, Bahadır E, Gülçiçek OB, Yiğitbaş H, Çelik A, Karagöz $A$, et al. Nesfatin-1 improves oxidative skin injury in normoglycemic or hyperglycemic rats. Peptides. 2016;78:1-10. doi: 10.1016/j.peptides.2015.12.006. [PubMed: 26829459].

30. Ozturk C, Oktay S, Yuksel M, Akakin D, Yarat A, Kasimay Cakir 0 . Anti-inflammatory effects of nesfatin-1 in rats with acetic acid-induced colitis and underlying mechanisms. J Physiol Pharmacol. 2015;66(5):741-50. [PubMed: 26579580].

31. Angelone T, Filice E, Pasqua T, Amodio N, Galluccio M, Montesanti G, et al. Nesfatin-1 as a novel cardiac peptide: identification, functional characterization, and protection against ischemia/reperfusion injury. Cell Mol Life Sci. 2013;70(3):495-509. doi: 10.1007/s00018-012-1138-7. [PubMed: 22955491].

32. Chiari PC, Bienengraeber MW, Pagel PS, Krolikowski JG, Kersten JR, Warltier DC. Isoflurane protects against myocardial infarction during early reperfusion by activation of phosphatidylinositol-3- kinase signal transduction: evidence for anesthetic-induced postconditioning in rabbits. Anesthesiology. 2005;102(1): 102-9. doi: 10.1097/00000542-200501000-00018. [PubMed: 15618793].

33. Mazurek T, Zhang L, Zalewski A, Mannion JD, Diehl JT, Arafat H, et al. Human epicardial adipose tissue is a source of inflammatory mediators. Circulation. 2003;108(20):2460-6. doi: 10.1161/01.CIR.0000099542.57313.C5. [PubMed: 14581396].

34. Baker AR, Silva NF, Quinn DW, Harte AL, Pagano D, Bonser RS, et al. Human epicardial adipose tissue expresses a pathogenic profile of adipocytokines in patients with cardiovascular disease. Cardiovasc Diabetol. 2006;5:1. doi: 10.1186/14752840-5-1. [PubMed: 16412224].

35. Eroglu S, Sade LE, Yildirir A, Bal U, Ozbicer S, Ozgul AS, et al. Epicardial adipose tissue thickness by echocardiography is a marker for the presence and severity of coronary artery disease. Nutr Metab Cardiovasc Dis. 2009;19(3):211-7. doi: 10.1016/j.numecd.2008.05.002. [PubMed: 18718744]. 